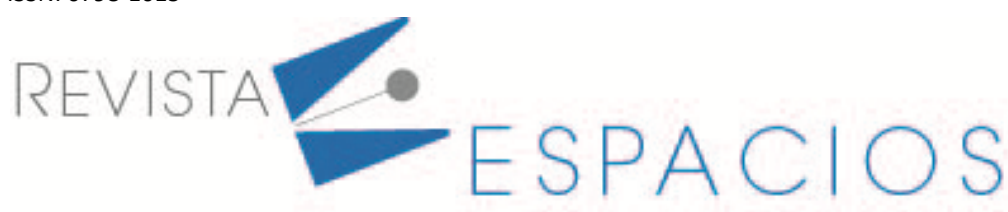

Vol. 41 (45) $2020 \cdot$ Art. 7

Recibido/Received: 12/06/2020 • Aprobado/Approved: 15/10/2020 • Publicado/Published: 26/11/2020

\title{
Impacto de la comercialización de futuros sobre el índice Colcap en la volatilidad asimétrica del mercado de acciones en Colombia
}

\section{Impact of the colcap index futures trading on the asymmetric volatility of the Colombian stock market}

RANGEL, Julián A. ${ }^{1}$

BLANDÓN, Alexander ${ }^{2}$

PINZÓN, José V. ${ }^{3}$

\begin{abstract}
Resumen
La pregunta motivadora de esta investigación fue medir el impacto sobre la volatilidad asimétrica en el mercado de acciones como consecuencia de la entrada en operación del mercado de futuros sobre el indicador bursátil COLCAP de la Bolsa de Valores de Colombia. Con base en modelos estadísticos del tipo GARCH, se analizaron los retornos diarios del indicador desde principios del 2008 hasta finales del año 2018. Los hallazgos revelan la existencia de un efecto de apalancamiento y un mayor nivel de asimetría después que los futuros comenzaron a ser comercializados.

Palabras clave: futuros de índice bursátil, volatilidad asimétrica, efecto de apalancamiento, GARCH.

Abstract

The motivatory question of this research was to measure the impact on the asymmetric volatility of the Colombian stock market as a consequence of the introduccion of the COLCAP index futures market in the Colombian Stock Exchange. Based on statistics models of the GARCH class, daily yields of the index were analyzed from the beginning of 2008 to the end of 2018. The findings show the existence of a leverage effect and a higher level of asymmetry after the futures began to be trading.

key words: stock index futures, asymmetric volatility, leverage effect, GARCH.
\end{abstract}

\section{Introducción}

Uno de los mercados más dinámicos e innovadores dentro del conjunto de mercados financieros, es el mercado de instrumentos derivados. A través de la negociación de forwards, futuros, opciones, swaps e instrumentos más complejos construidos a partir de estos derivados básicos, es posible diseñar estrategias para protegerse contra los principales riesgos que enfrentan los diferentes actores de los mercados financieros internacionales.

\footnotetext{
${ }^{1}$ M.Sc. in Finance. Profesor de la Facultad de Ciencias Económicas y Administrativas. Universidad del Tolima - UT. jarangel@ut.edu.co

2 Ph.D. in Development Studies. Profesor de la Facultad de Ciencias Económicas y Administrativas. Universidad del Tolima - UT. ablandonl@ut.edu.co

${ }^{3}$ Mag. en Administración. Profesor de la Facultad de Ciencias Económicas y Administrativas. Universidad del Tolima - UT. jvpinzon@ut.edu.co
} 
Riesgos como el movimiento adverso en las tasas de interés, la apreciación o depreciación de una divisa, el aumento o la disminución en el precio de mercado de un determinado bien o servicio, etc., pueden ser manejados y controlados muy eficientemente con la negociación de derivados financieros.

Por otra parte, la negociación de instrumentos derivados puede tener implicaciones sobre el comportamiento del mercado del activo subyacente. Por ejemplo, la comercialización de futuros u opciones sobre un determinado activo puede afectar variables características del mercado spot de este activo, como son su liquidez, su volatilidad, la velocidad de incorporación en los precios de nuevas noticias, etc. En particular, es posible que el mercado de derivados afecte la volatilidad asimétrica negativa o también conocida como efecto de apalancamiento del mercado spot del activo negociado, lo cual es precisamente el tema de esta investigación.

En el caso de la volatilidad, diversos investigadores han reportado impactos variados del mercado de derivados sobre la volatilidad del mercado del activo subyacente. Por ejemplo, en la investigación de Gulen y Mayhew (2000) se puede leer lo siguiente:

"Nuestros resultados indicaron que en los mayores dos mercados, los Estados Unidos y Japón, la volatilidad puede haberse incrementado después de listados los futuros sobre el índice accionario. Por otro lado, la volatilidad decreció o se mantuvo aproximadamente igual en la mayoría de los otros países de nuestra muestra, con decrecimientos estadísticamente significativos en muchos casos (p. 681)."

Fong y Han (2015) encontraron en una investigación sobre el mercado accionario de Hong Kong que con la entrada del mercado de futuros, la volatilidad del mercado spot disminuyó mientras que con la comercialización de opciones la volatilidad aumentó. Adicionalmente, estos autores también reportan la persistencia del efecto en la volatilidad debido a la negociación de los futuros y la disipación del impacto en la volatilidad provocado en el mercado subyacente por el mercado de opciones en la medida en que aumenta la madurez del mercado de derivados.

En otros países asiáticos los resultados sobre estos efectos también son mixtos. Por ejemplo, Cimen (2018) al estudiar el comportamiento del mercado accionario de Turquía desde 1997 hasta el 2015, detectó una significativa disminución de la volatilidad debido a la comercialización de futuros y opciones sobre un índice representativo del mercado accionario o de renta variable de ese país. Por su parte, Yao (2016) al analizar el impacto del mercado de futuros sobre el índice CSI 300 del mercado accionario Chino desde el año 2005 hasta el año 2015, encontró que después del lanzamiento del mercado de futuros, la volatilidad del mercado spot aumentó en forma considerable.

En cuanto al mercado de la India, uno de los más recientes estudios realizado por Joshi (2018) encontró que para las acciones de más alta capitalización de mercado, la volatilidad de las acciones aumentó considerablemente una vez listadas las opciones para estas acciones como activo subyacente. Para las acciones de media o pequeña capitalización, la disminución de la volatilidad fue estadísticamente insignificante. Finalmente, para el mercado de Tailandia, Bamrungsap (2018) utilizando datos del índice accionario SET50 desde el año 2000 hasta mediados del 2018, logra concluir que el mercado de futuros de Tailandia no induce la volatilidad de los precios spot y tampoco desestabiliza el mercado spot subyacente, sino que por el contrario, reduce la volatilidad del mercado accionario.

Ahora bien, la operación del mercado de derivados sobre acciones también puede inducir la aparición del efecto de apalancamiento en un mercado bursátil accionario sin registro alguno de su presencia o bien modificar sus rasgos y particularidades cuando el mismo es característico del comportamiento de su volatilidad. 
De esta manera, la interacción entre los mercados spot y los mercados de derivados, donde se negocian contratos que dependen del precio de estos activos subyacentes, inquieta a los inversionistas quienes buscan valorar los riesgos involucrados en la conformación de portafolios más diversificados y por supuesto más eficientes.

En síntesis, esta investigación pretende determinar el impacto de la comercialización de contratos de futuros sobre acciones en la volatilidad asimétrica negativa o efecto de apalancamiento del mercado de renta variable Colombiano. Para lograr este objetivo, se modelará el mercado de renta variable de la Bolsa de Valores de Colombia - BVC, debidamente segmentado por el inicio en esta misma Bolsa de la negociación de contratos de futuros sobre el índice accionario representativo del mercado.

Este estudio se justifica en la medida en la cual permite un mejor entendimiento de las variables que afectan la volatilidad de los mercados bursátiles, de tal manera que se puedan lograr portafolios de inversión más eficientes y diversificados y de esta manera promover el desarrollo y crecimiento de los mercados financieros y mejorar su regulación.

\section{Marco teórico}

El efecto de apalancamiento aparece mencionado por primera vez en un trabajo publicado por la Asociación Estadística Americana - ASA, por sus siglas en inglés, en el cual Black (1976) al estudiar el mercado accionario Americano, reporta que la volatilidad del precio de las acciones aumenta más cuando se presenta una repentina caída en los precios de las acciones que cuando se presenta una subida inesperada del mismo tamaño. Como posible explicación a este comportamiento, este autor señala que al disminuir el patrimonio de la firma, aumenta la razón de deuda sobre capital, lo cual se traduce en un mayor riesgo para la firma y por consiguiente en una más alta volatilidad.

Investigaciones posteriores confirmaron el efecto de apalancamiento o volatilidad asimétrica negativa en muchos mercados bursátiles de diferentes países. No obstante, la discusión sobre las reales causas de esta característica de los mercados continuó en los años siguientes. Así, Figlewski y Wang (2000) llegaron a la conclusión que existía muy poca evidencia para atribuir el efecto de apalancamiento al llamado apalancamiento financiero de la firma. En el mismo sentido, la correlación negativa entre el retorno de un activo y sus cambios de volatilidad son difíciles de explicar solamente por el cambio en la estructura de capital de la firma (Ait-Sahalia, Fan y Li, 2013; citados por Dahlvid y Granberg, 2017).

Adicionalmente, otros investigadores han cuestionado la explicación del apalancamiento financiero o variación de la razón de deuda sobre capital como el generador de la volatilidad asimétrica negativa al resaltar que este comportamiento no es exclusivo de los mercados de acciones, sino que por el contrario se puede presentar también en otros mercados como los de monedas, materias primas, metales, etc., en donde la composición entre deuda y patrimonio no es relevante. Por ejemplo, Maya y Gómez (2008) reportaron en una investigación sobre el comportamiento de las tasas de cambio frente al dólar y al euro de las monedas de 5 países Latinoamericanos con economías emergentes desde el año 2000 al 2007, que el real Brasilero y el sol Peruano presentaban volatilidad asimétrica (negativa en el primer caso y positiva en el segundo), mientras que el peso Colombiano, el peso Chileno y el peso Mexicano, mostraron una respuesta simétrica en la volatilidad frente a las aumentos o disminuciones del valor de la moneda.

En el mismo sentido, Wang y Yang (2009) hallan evidencia de volatilidad asimétrica en el valor diario de las monedas de algunos países desarrollados frente al dólar de Estados Unidos. Sin embargo, en este mismo estudio, la tasa de cambio del euro en términos del dólar americano mostró volatilidad simétrica ante apreciaciones o depreciaciones de la divisa de igual magnitud. Más recientemente, Panda, Nanda, Singh y Kumar (2019) examinan el mercado internacional de divisas de economías emergentes y en crecimiento después del 
año 2008 y encuentran un importante efecto de apalancamiento en el rublo Ruso y en la rupia de la India. Por otra parte, Dritsaki (2019) utilizando modelos econométricos asimétricos del tipo GARCH, estos últimos introducidos por Bollerslev (1986), reporta el efecto de apalancamiento en los retornos de la tasa de cambio del euro frente al dólar Americano.

Como puede observarse en los resultados de las investigaciones cuyos autores se han referenciado arriba, el efecto de apalancamiento en el mercado de divisas es un fenómeno frecuente que ha sido reportado para diferentes monedas y en diferentes períodos, no solamente de países desarrollados sino también de países con economías emergentes en todo el mundo.

En relación al efecto de apalancamiento en el mercado de materias primas o "commodities", Brooks y Prokopczuk (2013) citados por Chevallier y lelpo (2017), encontraron que la correlación entre los retornos y su volatilidad tiene un signo que es específico a cada mercado. En el caso del petróleo crudo, un retorno negativo grande en el precio de este combustible dispara un aumento en su volatilidad que es mayor que en el caso de un retorno positivo de similar magnitud. En el caso del oro, la plata y la soya, el comportamiento es todo lo contrario.

Por otra parte, Bampinas, Panagiotidis y Rouska (2019), realizaron un estudio para detectar una posible relación entre la actividad de busqueda de información en Google y la volatilidad de los mercados spot de petróleo y de oro. Estos investigadores examinaron datos desde el 2004 hasta el 2014, obtenidos de la Reserva Federal en el caso del oro y de la Agencia de Información de Energía de los Estados Unidos (EIA), en el caso del petróleo, para concluir que la demanda de información en Google por inversionistas no informados reduce la volatilidad asimétrica producida por impactos negativos sobre el precio en los mercados de estos dos commodities.

Otro estudio que analiza la volatilidad asimétrica en el mercado de commodities es el de Baur y Dimpfl (2018), quienes estudian los mercados spot de productos agrícolas, commodities de energía, metales industriales y metales preciosos, con un amplio horizonte de datos desde 1980 al 2016. Estos autores concluyen que efectivamente existe un efecto asimétrico positivo (un aumento de precios produce más volatilidad que una disminución de igual magnitud), que tiende a disminuir con el tiempo y a comportarse más como el efecto en los mercados de títulos de patrimonio o de capital.

Finalmente, Baum, Zerilli y Chen (2018), utilizando datos con una alta frecuencia, encuentran evidencia de apalancamiento para el mercado del petróleo crudo y de apalancamiento inverso, es decir, una volatilidad más alta cuando se incrementan los retornos, para el caso del mercado de gas.

Además de la teoría de Black (1976), otras teorías han tratado de encontrar los motivos por el cual se presenta la volatilidad asimétrica negativa en muchos mercados, sin embargo, persisten dudas sobre sus alcances y conclusiones. Dentro de estas teorías esta el efecto de retroalimentación de la volatilidad, el cual argumenta que un incremento anticipado en la volatilidad aumentaría la tasa de retorno requerida, lo cual a su vez provocaría una disminución del precio para aumentar los retornos futuros. Esta teoría ha sido estudiada por varios autores como Bollerslev, Litvinova y Tauchen (2006), Aboura y Wagner (2010) y más recientemente por Sun y Wu (2018).

Adicionalmente, algunos investigadores han encontrado que el efecto de apalancamiento para el caso de los retornos de las acciones de las empresas depende de las características propias de cada compañía. Por ejemplo, Voogd y Geschiere (2015) examinaron alrededor de 290 empresas Europeas desde el año 1999 hasta el 2014 y encontraron que el tamaño de la firma, el margen neto y la relación precio sobre ganancia, influyen considerablemente en el tamaño de las asimetrías de la volatilidad. Estos autores también confirmaron lo que ya habían señalado otros investigadores, que la relación de deuda sobre patrimonio, postulada por Black (1976) como la causa probable del efecto de apalancamiento, no afectaba en forma significativa el tamaño de la volatilidad asimétrica. 
Con respecto a la existencia del efecto de apalancamiento en los mercados accionarios del mundo, los resultados son tan variados como interesantes. Por ejemplo, en el Reino Unido, Butterworth (2000) analizó el efecto de la negociación de contratos de futuros sobre el índice FTSE mid 250 sobre el mercado spot subyacente, encontrando un comportamiento simétrico en la volatilidad. En Taiwan, Chiang y Wang (2002) encontraron evidencia de un cambio en la respuesta asimétrica en la volatilidad de los precios spot de las acciones debido a la negociación de contratos de futuros sobre índices bursátiles que miden la actividad de estos títulos. En Turquía, Caglayan (2011) analizó la respuesta del mercado accionario al lanzamiento del mercado de futuros sobre el índice ponderado accionario ISE30 de la Bolsa de Valores de Estambul desde el año 2002 al 2008, reportando que diferentes tipos de noticias de igual magnitud tenían el mismo impacto en la volatilidad antes de la negociación de contratos de futuros. Sin embargo, una vez este mercado de derivados entra en funcionamiento, las malas noticias incrementan más la volatilidad que las buenas noticias de la misma magnitud. En China, Xie y Huang (2014) concluyen, después de modelar las rentabilidades diarias producidas por los cambios del índice CSI 300 del mercado accionario del país desde el año 2005 al año 2012, que el lanzamiento de los futuros sobre el índice accionario no disminuyeron la volatilidad del mercado spot y tampoco provocaron la aparición de efecto de apalancamiento alguno, en un mercado que previamente tampoco registraba este tipo de comportamiento de su volatilidad. Finalmente, en Indonesia, Triady, Kurniasari, Utami y Sofian (2016) confirman la existencia del efecto de apalancamiento al examinar el mercado accionario del país entre los años 2001 y 2012, con un aumento considerable de este fenómeno en períodos de crisis.

Las anteriores referencias muestran que a nivel internacional es muy grande la preocupación de inversionistas, investigadores, académicos y reguladores por entender el comportamiento de los mercados de derivados y sus efectos sobre el mercado de activos subyacentes. Esta investigación se centra precisamente en explorar uno de esos efectos: el impacto del mercado de futuros sobre la volatilidad asimétrica del mercado accionario. Las investigaciones sobre este tema en Colombia son muy pocas, producto quizás de la incipiente etapa de desarrollo del mercado de derivados en la nación. Sin embargo, lograr portafolios de inversión más eficientes y diversificados y en general promover el desarrollo y crecimiento de los mercados financieros y mejorar su regulación, requiere necesariamente entender los variables que afectan la volatilidad de los mercados bursátiles del país.

\section{Metodología}

Los modelos GARCH - Generalized Autoregressive Conditional Heteroskedasticity- propuestos por Bollerslev (1986), estiman la varianza condicionada de procesos aleatorios como una función lineal de los rezagos de la varianza y de los cuadrados de los errores pasados. Por lo tanto, dado $R_{t}$ un proceso aleatorio descrito por:

$$
\mathrm{R}_{\mathrm{t}}=\delta_{0}+\sum_{\mathrm{i}=1}^{\mathrm{k}} \delta_{\mathrm{i}} \mathrm{R}_{\mathrm{t}-\mathrm{i}}+\varepsilon_{\mathrm{t}} \quad ; \quad \varepsilon_{\mathrm{t}} \sim \mathrm{N}\left(0, \sigma_{\mathrm{t}}^{2}\right)
$$

La varianza de los errores se puede representar entonces con un modelo $\operatorname{GARCH}(p, q)$ :

$$
\sigma_{\mathrm{t}}^{2}=\alpha_{0}+\sum_{\mathrm{i}=1}^{\mathrm{q}} \alpha_{\mathrm{i}} \varepsilon_{\mathrm{t}-\mathrm{i}}^{2}+\sum_{\mathrm{j}=1}^{\mathrm{p}} \beta_{\mathrm{j}} \sigma_{\mathrm{t}-\mathrm{j}}^{2}
$$

Las variables $\mathrm{R}_{\mathrm{t}-\mathrm{i}}$ en la ecuación [1] representan los rezagos de la variable. Además, se debe cumplir que $\alpha_{0}>$ $0, \alpha_{i} \geq 0, \beta_{j} \geq 0$ para que $\sigma_{t}^{2}$ sea mayor que cero en la ecuación [2]. La parte de la ecuación [2] que contiene los rezagos de la varianza $\sigma_{\mathrm{t}-\mathrm{j}}^{2}$, se denomina el término $\mathrm{GARCH}$, mientras que la parte con los cuadrados de los errores $\varepsilon_{\mathrm{t}-\mathrm{i}}^{2}$, se denomina el término $\mathrm{ARCH}$. El valor $\mathrm{p}$ representa la cantidad de rezagos de la varianza mientras que q la cantidad de rezagos de los errores. Además $\sum_{\mathrm{i}=1}^{\mathrm{q}} \alpha_{\mathrm{i}}+\sum_{\mathrm{j}=1}^{\mathrm{p}} \beta_{\mathrm{j}} \leq 1$ para asegurar la estacionariedad.

De acuerdo con Caglayan (2011), valores altos de los $\alpha_{i}$ significan que nueva información es incorporada más rapidamente a la varianza, similarmente, valores altos de $\beta_{\mathrm{j}}$ indican que la información vieja o más antigua es 
aún persistente.

Una de las aplicaciones más notables de los modelos GARCH es en el estudio de datos estadísticos a intervalos de tiempo regulares, en especial variables financieras que en no pocos casos presentan una volatilidad que cambia con el tiempo.

La Tabla 1 muestra un compendio de algunos de los más utilizados modelos de volatilidad condicional, los cuales fueron propuestos por diferentes autores a partir del modelo GARCH básico, con el objetivo de incorporar ciertas particularidades de las series de tiempo.

Una de las características del modelo GARCH es que la volatilidad es considerada como un parámetro simétrico, lo cual significa que el modelo es inadecuado para detectar el efecto de apalancamiento. Sin embargo, los otros modelos que se presentan en la Tabla 1 si permiten detectar diferencias en la volatilidad cuando los precios suben o bajan en la misma magnitud.

Tabla 1

Modelos GARCH

\begin{tabular}{ccc} 
Modelo & Ecuación de varianza & Autor \\
\hline $\operatorname{GARCH}(1,1)$ & $\sigma_{\mathrm{t}}^{2}=\alpha_{0}+\alpha_{1} \varepsilon_{\mathrm{t}-1}^{2}+\beta_{1} \sigma_{\mathrm{t}-1}^{2}$ & Bollerslev (1986) \\
$\operatorname{EGARCH}(1,1)$ & $\operatorname{Ln}\left(\sigma_{\mathrm{t}}^{2}\right)=\begin{array}{c}\alpha_{0}+\gamma\left(\frac{\varepsilon_{t-1}}{\sigma_{t-1}}\right)+\alpha\left(\frac{\left|\varepsilon_{t-1}\right|}{\sigma_{t-1}}-\left(\frac{2}{\pi}\right)^{0.5}\right) \\
+\beta \ln \left(\sigma_{t-1}^{2}\right)\end{array}$ & Nelson (1991) \\
$\operatorname{GJR}(1,1)$ & $\sigma_{\mathrm{t}}^{2}=\alpha_{0}+\alpha_{1} \varepsilon_{\mathrm{t}-1}^{2}+\beta_{1} \sigma_{\mathrm{t}-1}^{2}+\gamma \mathrm{D}_{\mathrm{t}-1}^{-} \varepsilon_{\mathrm{t}-1}^{2}$ & $\begin{array}{c}\text { Glosten, Jagannathan y } \\
\text { Runkle (1993) }\end{array}$ \\
$\operatorname{APARCH}(1,1)$ & $\sigma_{\mathrm{t}}^{2}=\alpha_{0}+\alpha_{1}\left(\left|\varepsilon_{\mathrm{t}-1}\right|-\gamma_{1} \varepsilon_{\mathrm{t}-1}\right)^{\delta}+\beta_{1} \sigma_{\mathrm{t}-1}^{\delta}$ & $\begin{array}{c}\text { Ding, Granger and Engle } \\
\text { (1993) }\end{array}$ \\
\hline
\end{tabular}

Esta tabla presenta el modelo GARCH básico y algunos de los más conocidos modelos con efecto de apalancamiento. La ecuación del modelo $\operatorname{GARCH}(1,1)$ incluye un término $\mathrm{GARCH}$ correspondiente a la parte que contiene el rezago de la varianza $\sigma_{\mathrm{t}-1}^{2} \mathrm{y}$ un término $\mathrm{ARCH}$ correspondiente a la parte que contiene el rezago de los cuadrados de los errores $\varepsilon_{\mathrm{t}-1}^{2}$. La ecuación del $\operatorname{EGARCH}(1,1)$ contiene el parámetro $\gamma$, el cual detecta el efecto de apalancamiento en los datos cuando su valor es significativo y diferente de cero. En el modelo $G J R(1,1)$, también conocido como GJR-GARCH $(1,1)$, cuando $\varepsilon_{\mathrm{t}-1}$ es negativo, la variable ficticia $\mathrm{D}_{\mathrm{t}-1}^{-}$vale 1 y cuando $\varepsilon_{\mathrm{t}-1}$ es mayor o igual a cero, $\mathrm{D}_{\mathrm{t}-1}^{-}$vale 0 . En la ecuación del modelo $\operatorname{APARCH}(1,1), \gamma_{1}$ es una medida del apalancamiento. Fuente: Caglayan (2011).

\section{Datos}

Los datos para la modelación se obtuvieron del índice accionario de capitalización COLCAP que calcula diariamente la Bolsa de Valores de Colombia - BVC, en el período comprendido entre enero 15 de 2008 y diciembre 28 de 2018. 
El índice COLCAP refleja las variaciones de los precios de las 20 acciones más líquidas que se negocian en el mercado Colombiano, donde el peso de cada acción esta determinado por el valor de capitalización bursátil ajustado de cada empresa. El índice comenzó a publicarse el 15 de Enero de 2008.

Teniendo en cuenta que en Febrero 28 del 2011 comenzó en la misma BVC la negociación de los futuros sobre el índice bursátil COLCAP, el período total se puede dividir en dos períodos consecutivos con el fin de detectar y medir la volatilidad asimétrica: un período inicial antes de la negociación de futuros que comprende del 15 de enero del 2008 hasta el 27 de febrero del 2011 y un período posterior que comienza con la negociación de futuros sobre el COLCAP en febrero 28 de 2011 y que se extiende hasta el 28 de diciembre de 2018.

Con base en los datos de cierre diarios del indicador COLCAP, la rentabilidad diaria compuesta continuamente se calculó utilizando la siguiente fórmula:

$$
\mathrm{R}_{\mathrm{t}}=\left[\ln (\text { Índice COLCAP } \mathrm{t})-\ln \left(\text { Índice } \operatorname{COLCAP}_{\mathrm{t}-1}\right)\right] * 100
$$

Donde Índice $\operatorname{COLCAP}_{t}$ corresponde al valor del indicador bursátil al final del día $t$.

A partir de la ecuación [3], para el período inicial antes de la negociación de futuros se obtuvieron 762 datos y para el período final, que comienza con la negociación de futuros sobre el COLCAP, se recolectaron 1,911 datos para un total de 2,673 datos o rentabilidades diarias en todo el período de estudio.

\section{Resultados}

Los siguientes son los resultados del análisis utilizando $\mathrm{R}$ como paquete estadístico. La Tabla 2 muestra los estadísticos descriptivos y las pruebas de normalidad, correlación y del multiplicador de Lagrange -LM de las tasas de retorno diarias del COLCAP, para los tres períodos definidos previamente: antes de la negociación de futuros sobre el índice accionario, posterior al comienzo de esta negociación y para el período completo.

Tabla 2

Estadísticos de la muestra y pruebas de normalidad, correlación y LM de los retornos diarios

\begin{tabular}{lccc}
$\begin{array}{c}\text { Estadístico Descriptivo / } \\
\text { Pruebas }\end{array}$ & $\begin{array}{c}\text { Período antes de la } \\
\text { negociación de futuros } \\
\text { (762 datos) }\end{array}$ & $\begin{array}{c}\text { Período que comienza con } \\
\text { la negociación de futuros } \\
\text { (1911 datos) }\end{array}$ & $\begin{array}{c}\text { Período total } \\
\text { (2673 datos) }\end{array}$ \\
\hline Media & 0.0706 & -0.0134 & 0.0105 \\
Desviación estándar & 1.3520 & 0.8778 & 1.0358 \\
Asimetría & -0.5399 & & \\
Exceso de Curtosis & 11.1048 & -0.1617 & -0.3632 \\
Jarque-Bera test & 2122.6 & 5.4430 & 11.1176 \\
Ljung-Box Q - test (1) & {$[0.0000]$} & 483.57 & 7398.1 \\
& 2.3148 & {$[0.0000]$} & {$[0.0000]$} \\
LM test (12) & {$[0.1281]$} & 37.272 & 26.945 \\
& 321.45 & {$[0.0000]$} & {$[0.0000]$} \\
& {$[0.0000]$} & 179.45 & 852.39 \\
& & {$[0.0000]$} & {$[0.0000]$} \\
\hline
\end{tabular}

Esta tabla presenta los estadísticos de la muestra y las pruebas Jarque-Bera, Ljung-Box y del multiplicador de Lagrange -LM para las tasas de rentabilidad diarias del indicador bursátil para tres períodos: un período inicial 
antes de la negociación de futuros que comprende desde el 15 de enero del 2008 hasta el 27 de febrero del 2011, un segundo período que comienza con la negociación de futuros sobre el COLCAP en febrero 28 de 2011 y que se extiende hasta el 28 de diciembre de 2018 y un tercer período o ciclo total conformado por estos dos períodos consecutivos anteriores. Las cifras entre corchetes [..] representan los valores de probabilidad. La prueba LjungBox-Pierce Q-test (1) verifica si los datos están independientemente distribuidos con base en la correlación serial hasta de primer orden. La prueba del multiplicador de Lagrange - LM test (12) busca heterocedasticidad condicional autorregresiva en los datos. Fuente: Elaboración Propia.

Como puede observarse en la Tabla 2, la desviación estándar para el período antes de la negociación de los contratos de futuros es de 1.3520 y de 0.8778 para el período posterior una vez comienza la comercialización de estos derivados. Esta disminución significa que los contratos de futuros no desestabilizaron el mercado de negociación de acciones en el país, al reducirse la volatilidad una vez estos comienzan a comercializarse. Con respecto a la asimetría, su valor negativo y pequeño para todos los períodos analizados muestran que las distribuciones de probabilidad de los retornos del índice accionario presentan valores más separados de la media a la izquierda que a la derecha. En cuanto al exceso de curtosis, sus valores superiores a 3.0 en todos los períodos indican leptocurtosis y colas pesadas.

La hipótesis nula de la prueba Jarque-Bera (1982) establece que los datos están normalmente distribuidos. En este caso, la hipótesis nula es violada debido a que los valores de probabilidad son significantes a un nivel del $1 \%$ antes y después del comienzo de la negociación de futuros y también para el ciclo completo.

La prueba Ljung-Box (1978) para la correlación serial hasta de primer orden permite verificar si los datos están independientemente distribuidos. Los estadísticos muestran que para el ciclo o período antes del comienzo de la operación del mercado de futuros, los datos efectivamente no presentan correlaciones. En cambio, para el ciclo que comienza con la negociación de estos derivados y para el ciclo completo se rechaza la hipótesis nula de datos independientes.

Finalmente, la prueba del multiplicador de Lagrange - LM test en la forma presentada por Silvey (1959), evidencian que la serie de tiempo presenta heterocedasticidad condicional autorregresiva o efectos ARCH cuando la misma considera hasta el rezago 12 para los tres ciclos en estudio, debido a que todos los estadísticos son significantes a un nivel del $1 \%$.

En conclusión, los estadísticos descriptivos y las pruebas de normalidad, correlación y LM de los retornos diarios del COLCAP permiten establecer en primer lugar, que para la modelación de los datos es conveniente utilizar una distribución diferente a la distribución normal, la cual permita manejar colas pesadas como las distribuciones $t$ (de Student) o de error generalizada GED y en segundo lugar, que es posible utilizar los modelos del tipo GARCH debido a que los mismos incorporan los efectos ARCH encontrados en las pruebas realizadas.

\section{Volatilidad asimétrica negativa en el mercado accionario}

Como se ha mencionado anteriormente, el efecto de apalancamiento en el mercado bursátil accionario aparece cuando la volatilidad del precio de las acciones se incrementa muchos más ante una repentina caída en los precios que ante una subida inesperada del mismo tamaño. Este efecto, también denominado como volatilidad asimétrica negativa, no es capturado por los modelos $\mathrm{GARCH}$, los cuales solo modelan una volatilidad simétrica (Dutta, 2014). Sin embargo, otros modelos desarrollados posteriormente al modelo GARCH básico, muy utilizados en simulaciones y que si detectan diferencias en la volatilidad cuando los precios suben o bajan en la misma magnitud, son los modelos de Glosten-Jagannathan-Runkle-GARCH, Exponential-GARCH y Asymetric Power ARCH, tal y como fue argumentado anteriormente. 
En la discusión de cual modelo es el más apropiado para realizar las simulaciones de los índices accionarios y sus características de volatilidad, no existe consenso en la comunidad de investigadores que den preferencia a uno u otro modelo, debido a que los resultados dependen de la condiciones particulares de cada mercado. Sin embargo, muchos autores si coinciden en la manera directa y sencilla como se pueden interpretar los resultados del Modelo de Glosten, Jagannathan y Runkle (1993). Este es el caso de Engle y Ng (1993) quienes concluyeron cuando estudiaron el mercado accionario Japonés que este modelo permitía capturar la varianza asimétrica con una mayor fidelidad. Adicionalmente, Kim y Kon (1994) muestran que el modelo de Glosten, Jagannathan y Runkle permite hacer una mejor descripción frente a otros modelos cuando se simula el comportamiento de títulos accionarios tomados por separado. Más recientemente, Bragoudakis y Voulgarakis (2019) al estudiar los mercados de divisas y de acciones de los países que conforman el llamado grupo BRICS - Brasil-Rusia-India-China y Sudáfrica, concluyeron que el modelo de Glosten, Jagannathan y Runkle capta mejor su comportamiento en comparación con otros modelos GARCH.

Considerando estas explicaciones y la deducción directa de resultados, esta investigación empleará el modelo de Glosten, Jagannathan y Runkle para detectar la volatilidad asimétrica mencionada.

De acuerdo con el modelo $G J R(1,1)$, la varianza en el período t se puede representar como:

$\sigma_{\mathrm{t}}^{2}=\omega+\alpha_{1} \varepsilon_{\mathrm{t}-1}^{2}+\beta_{1} \sigma_{\mathrm{t}-1}^{2}+\gamma D_{\mathrm{t}-1}^{-} \varepsilon_{\mathrm{t}-1}^{2}$

En la ecuación [4], cuando $\varepsilon_{\mathrm{t}-1}$ es negativo, la variable ficticia $\mathrm{D}_{\mathrm{t}-1}^{-}$vale 1 y cuando $\varepsilon_{\mathrm{t}-1}$ es mayor o igual a cero, $\mathrm{D}_{\mathrm{t}-1}^{-}$vale 0 . Bajo estas condiciones, aumentos repentinos en los precios o valores de error positivo producen un impacto $\alpha_{1}$ en la varianza, mientras que caídas bruscas en los precios o valores de error negativos producen un impacto $\alpha_{1}+\gamma$ en la varianza. Por supuesto, el efecto final dependerá del valor del coeficiente $\gamma$. Cuando este coeficiente es mayor que cero, el modelo ha detectado lo que se conoce como efecto de apalancamiento.

Por otra parte, de acuerdo con Caporin y Costola (2019), el modelo es estacionario cuando se cumple la siguiente condición:

$\gamma<2\left(1-\alpha_{1}-\beta_{1}\right)$

La rentabilidad del mercado accionario medida a través del comportamiento del índice bursátil fue representada con un modelo ARMA(n,m). Para determinar el número posible de términos autorregresivos $n$, se utilizó la prueba de la función de autocorrelación parcial. Adicionalmente, para determinar el número posible de términos de promedio móvil m, se utilizó la prueba de la función de autocorrelación serial. La cantidad posible de términos autorregresivos y de promedio móvil fueron de 0,1 y 4 en ambos casos.

La selección del mejor modelo para la media o rentabilidad y para la varianza se realizó buscando las menores probabilidades en la estimación de parámetros y los menores índices de los criterios de información Akaike (1974) y Bayesiano o Schwarz (1978) y el mayor índice de verosimilitud.

Teniendo en cuenta estos índices y la mejor estimación de parámetros, se encontró como mejor modelo la configuración ARMA $(1,0)$ - GARCH $(1,1)$ cuando se utiliza la distribución de probabilidad t (de Student) para las funciones de rentabilidad y de varianza aplicada a todo el horizonte de modelación. Como ya se ha mencionado, dado que el modelo GARCH no detecta la varianza asimétrica, se trabajó en su lugar con el Glosten, Jagannathan y Runkle GARCH(1,1) ó GJR(1,1), como también se conoce.

Las Tablas 3 y 4 muestran la estimación de coeficientes del modelo $\operatorname{ARMA}(1,0)$ - GJR(1,1) para los períodos antes y después de la comercialización de futuros respectivamente. Es de anotar que para las anteriores dos modelaciones, se verificó con la prueba Ljung-Box que los residuales no estuvieran correlacionados, es decir que 
fueran independientes, y con la prueba de Shapiro que no tuvieran una distribución normal, debido a que el modelo supone una distribución de probabilidad t (de Student).

Tabla 3

$\operatorname{ARMA}(1,0)-\operatorname{GJR}(1,1)$ antes de

la comercialización de futuros.

Estimación de Resultados

Ecuación de media ARMA(1,0):

$$
\begin{aligned}
& \mathrm{R}_{\mathrm{t}}=0.1025+0.0720 \mathrm{R}_{\mathrm{t}-1}+\varepsilon_{\mathrm{t}} \\
& \text { (0.0375) (0.0382) } \\
& \text { [0.0062] [0.0596] }
\end{aligned}
$$

Ecuación de varianza GJR(1,1):

$$
\begin{aligned}
& \sigma_{\mathrm{t}}^{2}=0.1606+0.0835 \varepsilon_{\mathrm{t}-1}^{2}+0.7048 \sigma_{\mathrm{t}-1}^{2}+0.1882 \mathrm{D}_{\mathrm{t}-1}^{-} \varepsilon_{\mathrm{t}-1}^{2} \\
& \begin{array}{llll}
(0.0513) & (0.0407) & \text { (0.0621) } & \text { (0.0749) }
\end{array} \\
& {\left[\begin{array}{llll}
{[0.0017]} & {[0.0400]} & {[0.0000]} & {[0.0119]}
\end{array}\right.}
\end{aligned}
$$

Estimación de coeficientes del modelo $\operatorname{ARMA}(1,0)$ - $G J R(1,1)$ para el período antes de la comercialización de futuros. Las cifras entre paréntesis (..) representan las desviaciones estándar y las cifras entre corchetes [..] los valores de probabilidad. Se utilizó una distribución de probabilidad $t$ (de Student). En la ecuación GJR(1,1): $\sigma_{\mathrm{t}}^{2}=$ $\alpha_{0}+\alpha_{1} \varepsilon_{\mathrm{t}-1}^{2}+\beta_{1} \sigma_{\mathrm{t}-1}^{2}+\gamma \mathrm{D}_{\mathrm{t}-1}^{-} \varepsilon_{\mathrm{t}-1}^{2}$, cuando $\varepsilon_{\mathrm{t}-1}$ es menor que 0 (disminuciones de precio), la variable $\mathrm{D}_{\mathrm{t}-1}^{-}$ toma el valor de 1 , por lo que la varianza es impactada en un valor $\alpha_{1}+\gamma$, mientras que si $\varepsilon_{\mathrm{t}-1}$ es mayor o igual a 0 (aumentos de precio), la variable $\mathrm{D}_{\mathrm{t}-1}^{-}$toma el valor de 0 , por lo que la varianza es impactada solamente en un valor $\alpha_{1}$. Fuente: Elaboración Propia.

\section{Tabla 4}

$\operatorname{ARMA}(1,0)$ - GJR(1,1) después de la comercialización de futuros.

Estimación de Resultados

Ecuación de media $\operatorname{ARMA}(1,0)$ :

$$
\begin{aligned}
\mathrm{R}_{\mathrm{t}}=0.004 & +0.1058 \mathrm{R}_{\mathrm{t}-1}+\varepsilon_{\mathrm{t}} \\
(0.0175) & (0.0236) \\
{[0.9788] } & {[0.0000] }
\end{aligned}
$$

Ecuación de varianza GJR(1,1):

$$
\begin{aligned}
& \sigma_{\mathrm{t}}^{2}=0.0300+0.0390 \varepsilon_{\mathrm{t}-1}^{2}+0.8512 \sigma_{\mathrm{t}-1}^{2}+0.1495 \mathrm{D}_{\mathrm{t}-1}^{-} \varepsilon_{\mathrm{t}-1}^{2} \\
& \begin{array}{llll}
(0.0120) & (0.0222) & (0.0389) & (0.0397)
\end{array} \\
& {\left[\begin{array}{llll}
{[0.0126]} & {[0.0784]} & {[0.0000]} & {[0.0001]}
\end{array}\right.}
\end{aligned}
$$

Estimación de coeficientes del modelo $\operatorname{ARMA}(1,0)$ - $G \mathrm{~J}(1,1)$ para el período después de la comercialización de futuros. Las cifras entre paréntesis (..) representan las desviaciones estándar y las cifras entre corchetes [..] los valores de probabilidad. Se utilizó una distribución de probabilidad t (de Student). Ecuación del modelo GJR(1,1): $\sigma_{\mathrm{t}}^{2}=\alpha_{0}+\alpha_{1} \varepsilon_{\mathrm{t}-1}^{2}+\beta_{1} \sigma_{\mathrm{t}-1}^{2}+\gamma \mathrm{D}_{\mathrm{t}-1}^{-} \varepsilon_{\mathrm{t}-1}^{2}$. Fuente: Elaboración Propia. 
Obsérvese que las dos modelaciones presentadas en las Tablas 3 y 4 son estacionarias, dado que se cumple la condición que $\gamma<2\left(1-\alpha_{1}-\beta_{1}\right)$. Adicionalmente, el coeficiente $\gamma$ es mayor que cero y significativo para los dos períodos analizados. En efecto, en los resultados de la Tabla 3 que muestran la modelación antes de la negociación de los futuros sobre el índice bursátil, se observa que el valor estimado del parámetro es de 0.1882 con un valor de probabilidad menor a 0.05 de significación. En la Tabla 4 para después del comienzo de la negociación de los futuros sobre el índice bursátil, se observa que el valor estimado del parámetro es de 0.1495 con un valor de probabilidad menor a 0.01 de significación. Los resultados anteriores implican que el modelo ha detectado el efecto de apalancamiento antes y después de la negociación de los futuros sobre el índice bursátil.

Con base en las modelaciones efectuadas, la Tabla 5 compara los niveles de asimetría para los períodos antes y después del lanzamiento del futuro sobre el índice COLCAP. Como puede observarse, el impacto sobre la volatilidad condicional de las noticias negativas es de 3.25 veces el impacto de las noticias positivas para el período antes de la negociación de los futuros sobre el índice bursátil. Este valor de asimetría aumenta a 4.83 para el período después de la negociación de los futuros sobre el índice.

Tabla 5

Comparación entre los niveles de asimetría para los períodos antes y después del c omienzo de la negociación de los futuros sobre el índice COLCAP en la BVC.

\begin{tabular}{ccc}
\hline $\begin{array}{c}\text { Variables y nivel de } \\
\text { asimetría }\end{array}$ & $\begin{array}{c}\text { Período antes de la } \\
\text { negociación de } \\
\text { futuros }\end{array}$ & $\begin{array}{c}\text { Período después de la } \\
\text { negociación de } \\
\text { futuros }\end{array}$ \\
\hline$\alpha_{1}$ & 0.0835 & 0.0390 \\
$\alpha_{1}+\gamma$ & 0.2717 & 0.1885 \\
$\alpha_{1}+\gamma$ & 3.2538 & 4.8333 \\
\hline
\end{tabular}

Esta tabla muestra el cálculo de los niveles de asimetría para el modelo $\mathrm{GJR}(1,1)$ con una distribución $\mathrm{t}$ (de Student). $\alpha_{1}$ representa el efecto de las noticias positivas sobre la volatilidad condicional, $\alpha_{1}+\gamma$ representa el efecto de las noticias negativas y $\left(\alpha_{1}+\gamma\right) / \alpha_{1}$ representa el nivel de asimetría. Fuente: Elaboración Propia

Como un segundo ejercicio de verificación, todas las simulaciones anteriores fueron adicionalmente efectuadas cambiando la distribución de probabilidad a una de error generalizada, con hallazgos parecidos a los aquí obtenidos.

En forma sucinta, es posible afirmar que antes de la negociación de futuros sobre el principal índice bursátil Colombiano, se presentaba un efecto de apalancamiento en el cual una noticia negativa generaba más fluctuaciones en los precios de las acciones que una noticia positiva de igual tamaño. Después de la introducción de este tipo de instrumento derivado, el efecto de apalancamiento continúa en el mercado accionario del país. Adicionalmente, el nivel de asimetría, el cual refleja en número de veces el mayor impacto sobre la volatilidad condicional de las noticias negativas con respecto a las noticias positivas, aumenta una vez entra en operación el mercado de futuros sobre el índice accionario.

\section{Conclusiones}

A nivel internacional es muy grande la preocupación de inversionistas, investigadores, académicos y reguladores por entender el comportamiento de los mercados de derivados y sus efectos sobre el mercado de activos subyacentes. Esta investigación se centra precisamente en explorar uno de esos efectos: el impacto del mercado de futuros sobre la volatilidad asimétrica negativa del mercado accionario. Las investigaciones sobre este tema en Colombia son muy pocas, producto quizás de la incipiente etapa de desarrollo del mercado de derivados en la nación. Sin embargo, lograr portafolios de inversión más eficientes y diversificados y en general promover el 
desarrollo y crecimiento de los mercados financieros y mejorar su regulación, requiere necesariamente entender las variables que afectan la volatilidad de los mercados bursátiles del país.

Con base en modelos estadísticos de la familia $\mathrm{GARCH}$, este estudio explora inicialmente la existencia de la volatilidad asimétrica en el mercado accionario Colombiano del 15 de enero del 2008 al 27 de febrero del 2011, período antes de la negociación de futuros sobre el COLCAP. Con el inicio de la negociación de estos derivados a partir del 28 de febrero del 2011, se estudia entonces como este mercado afecta la volatilidad del mercado spot accionario desde ese momento hasta el 31 de diciembre del 2018.

Los hallazgos revelan que antes de la negociación de futuros sobre el principal indicador bursátil colombiano, el mercado exhibía una volatilidad asimétrica negativa en el cual una noticia negativa generaba más fluctuaciones en los precios de las acciones que una noticia positiva de igual tamaño. Después de la introducción de este tipo de instrumento derivado, esta característica, también conocida como efecto de apalancamiento, persiste en el mercado de acciones del país; sin embargo, el nivel de asimetría, el cual mide el mayor impacto sobre la volatilidad condicional de las noticias negativas con respecto a las noticias positivas, es mayor comparado con el existente antes de la entrada en operación del mercado de los futuros sobre el índice accionario.

Por último y con respecto a la continuación de la investigación en este tema, es conveniente realizar otras aproximaciones diferentes a la modelación del tipo GARCH presentada en este documento, por ejemplo a través de los modelos de volatilidad estocástica o también utilizando los modelos TAR (Threshold Autoregressive Models), los cuales pueden contribuir a una mejor comprensión del comportamiento de la volatilidad asimétrica y su impacto en los mercados financieros nacionales e internacionales.

\section{Reconocimientos}

La investigación que da origen a este artículo ha sido posible gracias al apoyo y patrocinio de la Universidad del Tolima, Colombia, institución de educación superior con la cual los autores están profundamente agradecidos.

\section{Referencias bibliográficas}

Aboura, S. y Wagner, N. (2010). Extreme asymmetric volatility, leverage, feedback and asset prices. International Risk Management Conference. Florence, Italy. hal-01526073

Ait-Sahalia, Y., Fan, J. y Li, Y. (2013). The Leverage Effect Puzzle: Disentagling Sources of Bias at High Frequency. Journal of Financial Economics, Vol. 109, pp. 224-249.

Akaike, H. (1974). A new look at the statistical model identification. IEEE Transactions on Automatic Control. Vol. 19, No. 6. pp. 716-723.

Bampinas, G., Panagiotidis, T. y Rouska, C. (2019). The volatility persistence and asymmetry under the microscope: the role of information demand for gold and oil. Scottish Journal of Political Economy, Vol. 66, No. 1. pp. 180-197.

Bamrungsap, S. (2018). The impact of futures market on spot price volatility, and market efficiency: evidence from Thai stock index futures. Asian Administration \& Management Review, Vol. 1, No. 1, pp. 94-101.

Baum, C., Zerilli, P. y Chen, L. (2018). Stochastic volatility, jumps and leverage in energy and stock markets: evidence from high frequency data. Research Boston College Working Papers in Economics 952, Boston College Department of Economics.

Baur, D. y Dimpfl, T. (2018). The asymmetric return-volatility relationship of commodity prices. Energy Economics, Vol. 76, pp. 378-387. 
Bera, A. y Jarque, C. (1982); Model specification tests : a simultaneous approach. Journal of Econometrics, Vol. 20, No. 1, pp. 59-82.

Black, F. (1976). Studies of stock price volatility changes. Proceedings of the business and economics section of the American Statistical Association, pp. 177-181.

Bollerslev, T. (1986). Generalized autoregressive conditional heteroskedasticity. Journal of Econometrics, Vol. 31. pp. 307-327.

Bollerslev, T., Litvinova, J. y Tauchen, G. (2006). Leverage and volatility feedback effects in high-frequency data. Journal of Financial Econometrics, Vol. 4, No. 3. pp. 353-384.

Bragoudakis, Z. y Voulgarakis, R. (2019). A comparison of garch type models with the sv model on the financial markets of BRICS. SSRN Electronic Journal, Vol. 8, No. 19.

Brooks, C. y Prokopczuk, M. (2013). The dynamics of commodity prices. Quantitative Finance, Vol. 13, No. 4. pp. 527-542.

Butterworth, D. (2000). The impact of futures trading on underlying stock index volatility: the case of the FTSE Mid 250 contract. Applied Economics Letters, Vol. 7. pp. 439-442.

Caglayan, E. (2011). The impact of stock index futures on the Turkish spot market. Journal of Emerging Market Finance, Vol. 10. pp. 73-91.

Caporin, M. y Costola, M. (2019). Asymmetry and leverage in garch models: a news impact curve perspective. Applied Economics, Vol. 51. No. 31. pp. 3345-3364.

Chevallier, J. y lelpo, F. (2017). Investigating the leverage effect in commodity markets with a recursive estimation approach. Research in International Business and Finance, Vol. 39, parte B. pp. 763-778.

Chiang, M. and Wang C. (2002). The impact of futures trading on spot index volatility: evidence for Taiwan index futures. Applied Economics Letters, Vol. 9. pp. 381-385.

Cimen, A. (2018). The impact of derivatives on the volatility of the Turkish stock market. International Journal of Economic and Administrative Studies. Recuperado el 24 de Julio de 2019 de https://papers.ssrn.com/sol3/papers.cfm?abstract_id=3340904

Dahlvid, C. y Granberg, P. (2017). The leverage effect. Uncovering the true nature of U.S. asymmetric volatility. Recuperado el 5 de Noviembre de 2018 de http://lup.lub.lu.se/luur/download?func=downloadFile\&recordOld=8914682\&fileOld=8914688

Ding, Z., Granger, C. y Engle, R. (1993). A long memory property of stock market returns and a new model. Journal of Empirical Finance, Vol. 1, pp. 83-106.

Dritsaki, C. (2019). Modeling the volatility of exchange rate currency using garch model. International Economics, Vol. 72, No. 2. pp. 209-230.

Dutta, A. (2014). Modelling volatility: symmetric or asymmetric garch models? Journal of Statistics: Advances in Theory and Applications, Vol. 12, pp. 99-108.

Engle, R. y Ng, V. (1993). Measuring and testing the impact of news on volatility. Journal of Finance, Vol. 48, No. 5, pp. 1749-1778.

Figlewski, S. y Wang, X. (2000). Is the "leverage effect" a leverage effect? Unpublished working paper. Recuperado el 24 de Julio de 2019 de https://papers.ssrn.com/sol3/papers.cfm?abstract_id=256109 
Fong, L. y Han, C. (2015). Impacts of derivative markets on spot market volatility and their persistence. Applied economics, Vol. 47, No. 22, pp. 2250-2258. Recuperado el 24 de Julio de 2019 de http://www.tandfonline.com/10.1080/00036846.2015.1005813.

Glosten, L., Jagannathan, R. y Runkle, D. (1993). On the relation between the expected value and the volatility of the nominal excess return on stocks. Journal of Finance, Vol. 48, No. 5, pp. 1779-1801.

Gulen, H. y Mayhew S. (2000). Stock index futures trading and volatility in international equity markets. The Journal of Futures Markets, Vol. 20, No. 7, pp. 661-685.

Joshi, H. (2018). Does introduction of stock options impact stock volatility? empirical evidence from underlying stocks in Indian market. Theoretical Economics Letters, Vol. 8, pp. 1803-1815. Recuperado el 24 de julio de 2019 de https://www.scirp.org/journal/PaperInformation.aspx?PaperID=85371

Kim, D., Kon, S. (1994). Alternative models for the conditional heteroscedasticity of stock returns. The Journal of Business. Vol. 67, No. 4, pp. 563-598.

Ljung, G. y Box, G. (1978). On a measure of lack of fit in time series models. Biometrika. Vol. 65, No. 2, pp. 297303.

Maya, C. y Gómez, K. (2008). What exactly is 'bad news' in foreign exchange markets? Evidence from Latin American markets. Cuadernos de Economía, Vol. 45, pp. 161-183.

Nelson, D. (1991). Conditional heteroskedasticity in asset returns: a new approach. Econometrica, Vol. 59, No. 2, pp. 347-370.

Panda, A., Nanda, S., Singh, V. y Kumar, S. (2019). Evidence of leverage effects and volatility spillover among exchange rates of selected emerging and growth leading economies. Journal of Financial Economic policy, Vol. 11, No. 2. pp. 174-192.

Schwarz, G. (1978). Estimating the dimension of a model. The Annals of Statistics, Vol. 6, No. 2. pp. 461-464.

Silvey, S. (1959). The Lagrangian multiplier test. Annals of Mathematical Statistics. Vol. 30, No. 2, pp. 389-407.

Sun, Y. y Wu, X. (2018). Leverage and volatility feedback effects and conditional dependence index: a nonparametric study. Journal of Risk and Financial Management, Vol. 11, No. 2. pp. 1-20

Triady, M., Kurniasari, R., Utami, A. y Sofian, M. (2016). Investigation on leverage effect on Indonesian stock market. International Journal of Economics and Management, Vol. 10. pp. 1-17

Voogd, J. y Geschiere, T. (2015). Asymmetric volatility: testing firm-specific factors as a cause for the "leverage effect" using garch-modeling. Recuperado el 24 de Julio de 2019 de https://lup.lub.lu.se/studentpapers/search/publication/5472460

Wang, J. y Yang, M. (2009). Asymmetric volatility in the foreign exchange markets. Journal of International Financial Markets, Institutions and Money, Vol. 4, pp. 597-615.

Xie, S. y Huang J. (2014). The Impact of index futures on spot market volatility in China. Emerging Markets Finance and Trade, Vol. 50. pp. 167-177.

Yao, Y. (2016). The impact of stock index futures on spot market volatility. International Conference on Education, Sports, Arts and Management Engineering (ICESAME 2016). Recuperado el 24 de julio de 2019 de https://download.atlantis-press.com/article/25851103.pdf Esta obra está bajo una Licencia Creative Commons
Attribución-NoCommercial 4.0 International

(cc) BY-NC 